\title{
A integridade do endotélio e a terapia de reposição hormonal
}

\author{
Endothelial integrity and hormone replacement therapy
}

\section{Breve histórico}

As doenças cardiovasculares, com destaque para a doença coronariana isquêmica (DCI), são as principais causas de morbimortalidade na população com mais de 50 anos. As mulheres no menacme mantêm baixo risco de desenvolver DCI e este risco só irá aumentar significativamente no climatério, igualando-se ao homem em torno da sétima e oitava décadas de $v i d a^{1}$. Por ser de ocorrência rara no menacme, e o fato de mulheres ooforectomizadas ou após a menopausa terem aumento importante na incidência da DCI, foi sugerido, já há algum tempo, que o hipoestrogenismo estaria envolvido em sua etiologia. Por estas razões, o estudo da DCI passou a despertar interesse dos ginecologistas por estar diretamente relacionada às mudanças hormonais do climatério. Desta forma, a DCI poderia ser prevenida com terapia de reposição hormonal (TRH) e as suas variantes como a terapia hormonal (TH), terapia estrogênica (TE) ou terapia estroprogestativa (TEP).

Assim, no final do século passado, nas décadas de 1970 a 1990, muita ênfase foi dada para a TRH e sua prescrição aumentou, não só para corrigir os sintomas típicos da pósmenopausa, mas também como fator de proteção para várias doenças, inclusive e, principalmente, as doenças cardiovasculares.

De fato, os diversos estudos publicados naquele período, a maioria experimentais ou observacionais, sejam eles transversais ou longitudinais, coorte ou caso-controle, prospectivos ou retrospectivos e epidemiológicos suportavam, cientificamente, o uso em grande escala da TRH para mulheres climatéricas ${ }^{2-4}$. Embora não se tratasse de tema específico da Ginecologia, os eventos da especialidade tinham grandes espaços em sua carga horária dedicados ao assunto. E, obviamente, a indústria farmacêutica também tinha enorme interesse em patrociná-los e divulgá-los.

Evidentemente que a expansão da TH em todo o globo gerou uma expectativa para respostas concretas às frequentes indagações suscitadas pelas evidências acumuladas de estudos observacionais sobre a TH e doenças cardiovasculares (DCV), câncer de mama, osteoporose e outros tipos de doenças. Foi percebida a necessidade de estudos experimentais controlados, randomizados (clinical trials), com grande volume de pacientes, para responder a muitos questionamentos. É fácil se prever as dificuldades para a realização de estudos desta natureza, com grandes casuísticas. As dificuldades começam por seu financiamento, esbarrando nos

Correspondência:

Marcos Felipe Silva de Sá Departamento de Ginecologia e Obstetríci Avenida Bandeirantes, 3.900 CEP 14049-900 - Ribeirão Preto (SP), Brasil Fone: (16) 3602-2583 E-mail: marcosfelipe@fmrp.usp.br 
processos de seleção de pacientes e na necessidade de se organizarem múltiplos centros de pesquisa sob uma coordenação única.

Ao final dos anos 1990 e início dos anos 2000 vieram as publicações de alguns grandes trials, nos quais foram estudados os efeitos da TRH (TEP e TE) sobre a proteção primária ou secundária contra DCV. É possível citar o estudo Women's Health Iniciative (WHI) e o Heart and Estrogen/Progestin Replacement Study (HERS), , entre outros. Estes trials tiveram grande impacto sobre os conceitos vigentes em TRH, pois seus resultados mostraram que, em termos de prevenção primária, a associação entre estrogênios equinos conjugados (EEC) e acetato de medroxiprogesterona (AMP) aumentou o risco de doença arterial coronariana (DAC) e acidente vascular cerebral (AVC) em mulheres climatéricas pós-menopausa, quando comparadas com aquelas que usaram placebo ${ }^{5}$. O grupo de pacientes que usou exclusivamente EEC não apresentou aumento desta incidência quando comparado com as usuárias de placebo, embora mantivesse um aumento do risco de $\mathrm{AVC}^{7}$.

No estudo HERS, que inclusive antecedeu ao WHI, foram avaliadas mulheres que já haviam sofrido algum evento cardiovascular e, nestas, os resultados mostraram que a associação EEC+AMP aumentava o risco de DAC no primeiro ano de uso, não conferindo às pacientes qualquer proteção (prevenção secundária) .

A divulgação destes resultados refletiu sobre a prescrição hormonal de maneira bastante significativa. Nos Estados Unidos, por exemplo, a prescrição de TRH que crescia a taxas de 15\% ao ano, atingindo 90 milhões de prescrições em 1999, caiu para 57 milhões em 2003.

$\mathrm{O}$ que mais intrigou os pesquisadores foi que os resultados, tanto do WHI como do estudo HERS, representaram um contraponto aos conhecimentos até então vigentes e muitos outros questionamentos vieram à tona. Ambos os trabalhos foram publicados em revistas de grande impacto e não havia qualquer dúvida sobre a competência dos pesquisadores envolvidos. O WHI foi patrocinado com recursos governamentais e o HERS pela própria indústria farmacêutica, não havendo, portanto, nenhum interesse dos patrocinadores em induzir aos resultados negativos nos estudos em questão.

\section{A visão dos fatos nos dias de hoje}

Por que a diferença dos efeitos dos estrogênios quando comparados os estudos experimentais e observacionais com os trials? Muitas análises posteriores à divulgação dos resultados do WHI e HERS induziram a reflexões sobre os critérios de seleção das pacientes, levando especialmente a questionamentos, entre tantos outros, com relação às idades das pacientes, as quais são consideradas muito avançadas para os padrões de mulheres que corriqueiramente utilizam a TRH.

Por que a idade tem sido considerada tão importante assim? Os conhecimentos atuais parecem apontar que a diferença pode estar na maior ou menor integridade do endotélio vascular, a depender da faixa etária em consideração. No texto a seguir apresentaremos, de maneira bastante simplificada, algumas informações que poderão facilitar aos colegas ginecologistas um melhor entendimento desta relação entre a TH no climatério e os riscos para as DCV. Não há pretensão do editorialista em enfocar, com detalhes, os complexos mecanismos da função endotelial.

Até recentemente pouca importância era dada ao endotélio vascular como tecido ativo no organismo. Entretanto, a imensa malha vascular do corpo humano representa uma grande quantidade de células endoteliais contidas na superfície interna dos vasos. Para exemplificar, se as células endoteliais fossem dispostas lado a lado, cobririam uma superfície equivalente a seis quadras de tênis (de 4.000 a $7.000 \mathrm{~m}^{2}$ ) ou, caso fossem colocadas em linha teriam um comprimento equivalente a quatro voltas no planeta Terra ${ }^{8}$.

Apesar de possuírem as mesmas funções, as células endoteliais podem exercê-las de maneiras diferentes a depender dos estímulos do microambiente. Essa heterogeneidade é determinada geneticamente e por fatores ambientais. O endotélio tem como funções principais o controle do tônus vasomotor; a manutenção do sangue no estado fluído (hemostasia); o controle do fluxo de nutrientes e células e da permeabilidade vascular; a resposta inflamatória e a promoção da angiogênese ${ }^{9}$. Tendo em vista o tema em questão, vamos nos concentrar nas duas primeiras: o controle do tônus vasomotor e a hemostasia.

Para o controle do tônus vascular, o endotélio secreta tanto substâncias vasodilatadoras como o potente óxido nítrico (NO), prostaciclinas (PGI2), o EDHF (fator hiperpolarizante derivado do endotélio), substâncias vasoconstritoras como as endotelinas, tromboxane (TXA-2) e o fator ativador plaquetário (PAF). Em condições fisiológicas, o endotélio normal e sadio exerce com mais competência a função de promover a vasodilatação. Já o endotélio inflamado, acometido com placas ateromatosas secreta predominantemente substâncias vasoconstritoras.

No que diz respeito ao controle da hemostasia, as células endoteliais são capazes de sintetizar moléculas tromborreguladoras que inibem ou facilitam a formação do trombo, a depender do estímulo recebido por essas células. Como 
ação antitrombótica, exerce atividades antiplaquetárias importantes através da síntese de antiagregantes plaquetários como NO, PGI2 e adenosina. Além disso, apresenta atividades anticoagulantes facilitando a inibição dos fatores de coagulação como a antitrombina, a ativação da proteína $C$, a síntese da proteína $S$ e a síntese do inibidor da via do fator tecidual (TFPI) que inibe o fator tecidual (TF). A atividade fibrinolítica é exercida pela produção de ativadores do plasminogênio (tPA e uPA). Portanto, em condições fisiológicas, prevalecem as propriedades antitrombóticas do endotélio. Por outro lado, o endotélio doente e lesado favorece a secreção de fator tissular e a agregação plaquetária.

É sabido, também, que existem receptores para estrogênio e progesterona em todas as camadas dos vasos, particularmente na musculatura lisa. Artérias sadias expressam receptores de E2 com muita intensidade, e quando envelhecem ou se tornam ateroscleróticas vão perdendo esta expressão ${ }^{10}$. Os estrogênios exercem efeitos sobre os vasos de forma direta e indireta, eles diretamente induzem a síntese de NO (vasodilatador potente), reduzem a oxidação do LDL e das moléculas de adesão e migração de monócitos. Tem efeito pró-fibrinolítico reduzindo o PAI-1, mas atuam como pró-coagulantes reduzindo a proteína $\mathrm{S}$ e causando resistência à ação da proteína $\mathrm{C}$. Estes indiretamente atuam sobre os vasos, aumentando os níveis de HDL e reduzindo LDL e a homocisteína. Por outro lado, têm efeito pró-coagulante aumentando alguns fatores de coagulação.

Já os progestagênios têm menor influência sobre o endotélio, não alteram a coagulação ou a oxidação do LDL promovida pelo estradiol diretamente. Alguns autores apontam que os progestagênios reduzem a dilatação endotelial promovida pelo estradiol (não há consenso). Indiretamente podem atuar reduzindo o HDL e aumentando o LDL, reduzindo os triglicerídeos. Portanto, em menor escala, tendem a contrapor-se aos efeitos benéficos dos estrogênios, mas esta ação não é consensual ${ }^{11-13}$.

Assim, em condições fisiológicas, os estrogênios promovem vasodilatação via endotélio e protegem o endotélio contra a placa ateromatosa. Talvez estas ações possam explicar a baixa incidência de eventos cardiovasculares encontrados em mulheres jovens com bons níveis de estrogênios. Entretanto, à medida que a idade avança, o endotélio vai se tornando comprometido com a formação de placas ateromatosas e com processos inflamatórios em curso. As suas funções fisiológicas de promoção da vasodilatação e antitrombóticas são perdidas e passam a predominar as ações das substâncias que induzem a vasoconstrição e pró-trombóticas. Vários fatores contribuem para esta deterioração, como a dislipidemia, a hipertensão arterial sistêmica, o tabagismo, o diabetes mellitus, a hiper-homocisteinemia, a hereditariedade, o bioenvelhecimento e os baixos níveis de estrogênios encontrados após a menopausa.

Estes fatores predisponentes de risco aumentam o estresse oxidativo comprometendo as células endoteliais. Em um primeiro momento este comprometimento é apenas funcional, decorrente do persistente processo inflamatório local, mas, com o passar do tempo vão ocorrer alterações estruturais nos vasos e estas lesões facilitarão os fenômenos tromboembólicos que clinicamente se traduzem como infarto do miocárdio, AVC e outros eventos isquêmicos.

Estes fenômenos, evidentemente, não ocorrem somente por causa da instauração da menopausa. Esta corresponde a apenas a um divisor de águas nesta cronologia, pois incide numa idade que marca a transição entre diferentes fases da vida da mulher. Neste período, além da mulher perder a proteção estrogênica, o bioenvelhecimento, aliado a outros fatores predisponentes de riscos, agride o endotélio, levando à formação das placas ateromatosas acompanhadas do processo inflamatório com todas as suas consequências.

Já que a ação dos esteroides sexuais seria dependente da qualidade do endotélio, haveria então uma hora ideal de intervir com a TRH? Os dados apresentados pelos grandes trials, como o WHI, apontam nesta direção. Vejamos os fatos e os números deste estudo sob duas ópticas diferentes.

As conclusões dos autores da primeira publicação do WHI, em 2002, foram as seguintes: "os riscos à saúde excedem os benefícios após o uso de estrogênios + progestagênios em mulheres norte-americanas seguidas por 5,2 anos em média. A mortalidade geral não foi afetada. O risco 'benefício' encontrado neste trial não é consistente com o seu uso para prevenção primária de doenças crônicas, e os resultados indicam que este regime não deveria ser instituído ou continuado para prevenção primária de DCV (DAC)" 5 .

Uma das maiores críticas que este estudo recebeu foi exatamente a idade avançada das pacientes envolvidas, em média 63 anos (apenas 1/3 delas tinha menos de 60 anos e foram selecionadas mulheres até os 79 anos de idade). $\mathrm{O}$ tempo médio decorrido após a menopausa foi próximo de 15 anos e foram incluídas algumas mulheres diabéticas e hipertensas, com um grande número de obesas. Inúmeras publicações vieram à tona com críticas aos critérios de seleção das pacientes envolvidas no WHI, especialmente no que diz respeito à faixa etária.

Ora, neste momento da vida, a rede vascular das mulheres já sofreu severas alterações, não só funcionais, mas também estruturais decorrentes do bioenvelhecimento e da agressão dos diferentes fatores adversos já citados. Para demonstrar que aquelas conclusões dos autores do WHI estavam equivocadas, poderíamos citar uma publicação de Lobo $^{14}$, o qual analisou dois grandes trials - HOPE e Menopausal Study Group - realizados entre os anos de 1989 e 
2001. Em sua análise foram incluídas somente as pacientes brancas, saudáveis, com até $35 \%$ de desvio do peso ideal, pressão arterial menor que $160 \times 90 \mathrm{mmHg}$, colesterol $<300 \mathrm{mg} / \mathrm{dL}$, triglicérides $<300 \mathrm{mg} / \mathrm{dL}$, glicemia $<125 \mathrm{mg} / \mathrm{dL}$, tempo de menopausa com média de 4,9 anos, idade média de 53,6 anos. Foram excluídas as pacientes com antecedentes de câncer, trombose e DCV. Os resultados envolvendo 2.173 tratadas com 0,625 mg, EEC, com ou sem AMP por um ano, comparadas com o Grupo Placebo mostraram que o número de eventos cardiovasculares/1.000 mulheres/ano nas mulheres tratadas com TRH foi de 1,96 contra 3,01 no Grupo Placebo. Lobo concluiu que "a análise da combinação de evidências de dois grandes estudos, representando a experiência de coortes similares de 4.065 mulheres saudáveis e mais jovens na pós-menopausa, revelou a baixa incidência de doença cardíaca coronariana e outros eventos cardiovasculares dentro do primeiro ano de TH. Estes resultados, obtidos com 1,5 vezes o número de mulheres do WHI em faixa etária de 50 a 60 anos, contrasta marcadamente com os resultados daquele estudo. Importante lembrar que as mulheres do WHI não são representativas da maioria das mulheres que iniciam TH por causa dos sintomas próprios do climatério (isto é, mais próximo da menopausa, mais jovens e mais saudáveis)".

Esta e outras publicações levaram os próprios autores do WHI a reflexões sobre a forma de apresentação dos dados obtidos, uma vez que nas primeiras publicações deste megaestudo os números se referiram a todo o conjunto das mulheres, sem levar em conta a faixa etária e o tempo de menopausa.

Assim, em 2007 foram publicados os resultados dos mesmos dados, porém estratificando-os por faixa etária e considerando o tempo de menopausa e a suas interpretações sofreram modificações substanciais. Os números mostraram que, em mulheres com menos de 20 anos de menopausa, o risco relativo (RR) de doença cardíaca coronariana para as usuárias de TRH não foi estatisticamente diferente do grupo usuário de placebo. Com menos de dez anos de menopausa, houve seis casos a menos por 10.000 mulheres do que o esperado. Apenas após os 20 anos de menopausa os números mostraram risco significativamente maior $(\mathrm{RR}=1,28$; $\mathrm{IC}=1,03-1,58)$, com 17 casos a mais do que o esperado ${ }^{15}$.

Quando se analisou por idade, os dados também foram bastante diferentes do artigo original. Somente após os 70 anos de idade a TRH mostrou RR aumentado ( $\mathrm{R}=1,26$; $\mathrm{IC}=1,0-1,59)$ para doença cardíaca coronariana, com 19 casos adicionais/10.000 mulheres. Nas mulheres até 69 anos de idade não houve aumento do RR. A taxa de mortalidade em mulheres entre 50 e 60 anos teve o $R R=0,7$, e não apresentou aumento nas outras faixas etárias nas usuárias de TRH. Entretanto, a TH aumentou o risco de AVC, sem variação por idade ou tempo de menopausa $(\mathrm{RR}=1,32$; IC $=1,12-1,56)$. Os autores concluíram que, "embora não significativa, esta análise secundária sugere que o efeito dos hormônios sobre a doença cardíaca coronariana pode ser modificado pelos anos após a menopausa, com os maiores riscos em mulheres com 20 ou mais anos desde a menopausa (ou idade acima de 70 anos). As doenças cardíacas coronarianas tendem a ser reduzidas (não significativamente) em mulheres mais jovens ou mulheres com menos de dez anos desde a menopausa e o risco de mortalidade total foi reduzido em mulheres com idade entre 50 e 59 anos". "Uma tendência similar foi observada para a mortalidade total, mas os riscos para AVC foram mais elevados, independente dos anos após a menopausa" ${ }^{15}$.

Ainda, considerando pacientes do WHI e envolvendo o grupo tratado apenas com EEC, os autores do WHI analisaram 1.064 mulheres randomizadas, com idades entre 50 a 59 anos, média de 7,4 anos de tratamento e avaliadas cerca de 1,3 anos após o término do trial e avaliadas após 8,7 anos da randomização ${ }^{16}$. As pacientes foram submetidas à tomografia computadorizada do coração, tiveram o cálcio depositado nas artérias coronarianas e este foi medido pelo escore Agatston. O escore de Agatston é baseado nas características e dimensões da placa de calcificação. Quanto maior o escore, piores são as características da placa. Os resultados foram surpreendentes. As pacientes que tiveram o pior escore foram as que mais se beneficiaram da TH. A prevalência entre as expostas à TH comparadas com placebo variou conforme o escore Agatston. Para o escore 0, a Odds Ratio (OR) foi de 0,78 (IC=0,58-1,04); para o escore 10, $\mathrm{OR}=0,74(\mathrm{IC}=0,55-0,99)$; para o escore $100, \mathrm{OR}=0,69(\mathrm{IC}=0,48-0,98)$ e para o escore 300, $\mathrm{OR}=0,58(\mathrm{p}=0,03)$.

Considerando apenas as pacientes que tiveram mais de $80 \%$ de aderência ao tratamento, ou seja, as que mais sofreram influência da TH, a OR tem números mais significativos. Para o escore $0, \mathrm{OR}=0,64(\mathrm{p}=0,01)$; escore 10 , $\mathrm{OR}=0,55(\mathrm{p}<0,001)$; escore 100, $\mathrm{OR}=0,46(\mathrm{p}=0,001)$ e para o escore $300, \mathrm{OR}=0,39(\mathrm{p}=0,004)$.

Portanto, aquelas discrepâncias entre resultados de estudos observacionais e os estudos controlados randomizados se atenuaram. Esta nova análise dos dados do WHI aponta para algumas convergências entre eles, especialmente com os estudos observacionais em animais e laboratoriais os quais tiveram seu foco principalmente no efeito dos estrogênios sobre as artérias coronarianas normais e em mulheres sem evidências clínicas de DCV.

Desta forma, a idade das pacientes e o tempo decorrido após a menopausa são fatores extremamente importantes para determinar os riscos da TH nas mulheres climatéricas. Existe um momento mais favorável para o seu início, chamado de "janela de oportunidade" que corresponde ao período mais precoce do climatério, mais precisamente a partir do período perimenopáusico, quando os níveis de estrogênios estão decrescendo e a sintomatologia é mais exuberante, 
com forte predomínio dos sintomas vasomotores. Neste momento, os vasos apresentam menor comprometimento estrutural do seu endotélio e nestas condições os riscos cardiovasculares para a TE são minimizados. A mulher sintomática poderia se beneficiar da TE com maior segurança.

Por conta destas novas perspectivas, o endotélio passou a ser o alvo das pesquisas que têm buscado encontrar marcadores da sua função. No entanto, até o momento são encontradas inúmeras dificuldades. Algumas substâncias exercem apenas efeitos locais, sendo difícil detectar alterações na concentração sérica. Alguns marcadores séricos da função endotelial têm sido tentados, como o NO, o PAI-1, as moléculas de adesão, a endotelina, o PGI2, entre outros, mas seu uso ainda tem se restringido às pesquisas.

Alguns métodos mais invasivos têm sido utilizados, como a angiografia com infusão de agonistas vasodilatadores e a pletismografia, além da tonometria vascular. Mais recentemente está ganhando muitos adeptos a ultrassonografia da artéria braquial com medida da dilatação de fluxo - DILA - que é um método relativamente simples, não invasivo embora dependente do operador ${ }^{17}$.

Assim, a médio prazo será possível avaliar endotélio previamente à prescrição de TRH e a partir daí se poderia monitorizar o endotélio de pacientes de maior risco. Certamente advirão métodos de biologia molecular para rastrear disfunção endotelial, assim como para a avaliação genética de disfunções endoteliais herdadas.

As pesquisas deverão continuar e é previsto que as discussões sobre a TH serão voltadas para o papel dos progestagênios, considerando que os vários estudos controlados mostraram um efeito negativo da TH sobre as mamas e aparelho cardiovascular quando eles estão associados aos estrogênios. Velhos conceitos poderão cair por terra, uma vez que o uso tradicional de progestagênios derivados da $17 \mathrm{OH}$ progesterona (naturais), como é o caso da AMP, não se mostrou terapia segura. $\mathrm{O}$ advento de novos progestagênios, mesmo que derivados da nortestosterona, apesar do maior efeito androgênico, poderão se mostrar mais eficazes e de menor risco do que a AMP e, portanto, poderão ter seu lugar na TH do climatério.

As doses dos estrogênios a serem utilizadas estão sendo motivos de novos estudos, mas no momento ainda carecemos de resultados concretos para suportar o seu uso em baixas doses com a mesma eficácia, porém com redução dos riscos.

Seguindo nesta linha de raciocínio, a Sociedade Brasileira de Climatério em associação com a Sociedade Brasileira de Cardiologia debateram longamente esta questão e estabeleceram algumas diretrizes para a TRH ${ }^{18}$. Segundo aquelas diretrizes, a TRH não está recomendada com a finalidade exclusiva de reduzir o risco de DCV; existem evidências de benefícios cardiovasculares quando é iniciada nos primeiros anos de pós-menopausa - janela de oportunidade - e de riscos cardiovasculares, quando iniciada tardiamente; não existem estudos especificamente delineados com desfecho final para DCV em usuárias de TE ou TEP abaixo dos 60 anos; a TRH compreende inúmeras possibilidades de formulações com diferentes E, P e A, em diferentes regimes, doses e vias de administração; cada um dos hormônios empregados em TRH tem efeito próprio e singular, que pode variar na dependência de estar associado a outros hormônios; não se pode falar de "efeito de classe" da TRH sobre o risco de DCV. É preciso especificar regime, dose e via utilizados; e que não existem estudos em DCV com desfecho final para TRH de baixa dosagem ou tibolona.

Concluímos que a TRH, embora discutida calorosamente, ainda tem o seu espaço no arsenal terapêutico. Mas, é preciso respeitar as indicações, hoje restritas às pacientes sintomáticas e, sobretudo, as suas contraindicações e efeitos colaterais. O seu início deve-se dar preferencialmente no momento mais oportuno, o qual é chamado de "janela de oportunidades".

\section{Referências}

1. Rosano GM, Panina G. Cardiovascular pharmacology of hormone replacement therapy. Drugs Aging. 1999;15(3):219-34.

2. Grodstein F, Stampfer M, Manson JE, Colditz GA, Willett WC, Rosner B, et al. Postmenopausal estrogen and progestin use and the risk of cardiovascular disease. N Engl J Med. 1996;335(7):453-61.

3. Stampfer M, Colditz GA, Willet WC, Manson JE, Rosner B, Speizer FE, et al. Postmenopausal estrogen therapy and cardiovascular disease. Ten-year follow-up from the nurses's health study. N Engl J Med. 1991;325(1 1):756-62.

4. Herrington DM, Reboussin DM, Brosnihan KB, Sharp PC, Shumaker SA, Snyder TE, et al. Effects of estrogen replacement on the progression of coronary-artery atherosclerosis. N Engl J Med. 2000;343(8):522-9.

5. Rossouw JE, Anderson GL, Prentice RL, LaCroix AZ, Kooperberg C, Stefanick ML, et al. Risks and benefits of estrogen plus progestin in healthy postmenopausal women: principal results from Women's Health Initiative randomized controlled trial. JAMA. 2002;288(3):321-33. 
6. Hulley S, Grady D, Bush T, Furberg C, Herrington D, Riggs B, et al. Randomized trial of estrogen plus progestin for secondary prevention of coronary heart disease in postmenopausal women. Heart Estroge/Progestin Replacement Study (HERS) Research Group. JAMA. 1998;280(7):605-13.

7. Anderson GL, Limacher M, Assaf AR, Bassford T, Beresford SA, Black H, et al. Effects of conjugate equine estrogen in postmenopausal women with hysterectomy: the Women's Health Initiative randomized controlled trial. JAMA. 2004;291(14):1701-12.

8. Wolinsky H. A proposal linking clearance of circulating lipoproteins to tissue metabolic activity as a basis for understanding atherogenesis. Circ Res. 1980;47(3):301-11.

9. Cines DB, Pollak ES, Buck CA, Loscalzo J, Zimmerman GA, McEver RP, et al. Endothelial cells in physiology and in the pathophysiology of vascular disorders. Blood. 1998;91(10):3527-61.

10. Losordo DW, Kearney M, Kim EA, Jekanowski J, Isner JM. Variable expression of the estrogen receptor in normal and atherosclerotic coronary arteries of premenopausal women. Circulation. 1994;89(4):1501-10.

11. Wakatsuki A, Okatani Y, Ikenoue N, Fukaya T. Effect of medroxyprogesterone acetate on endothelium-dependent vasodilation in postmenopausal women receiving estrogen. Circulation. 2001;104(15):1773-8.

12. Girdler SS, Hinderliter AL, Wells EC, Sherwood A, Grewen KM, Light KC. Transdermal versus oral estrogen therapy in postmenopausal smokers: hemodynamic and endothelial effects. Obstet Gynecol. 2004;103(1):169-80.

13. Gerhard M, Walsh BW, Tawakol A, Haley EA, Creager SJ, Seely EW, et al. Estradiol therapy combined with progesterone and endotheliumdependent vasodilation in postmenopausal women. Circulation. 1998;98(12):1158-63.

14. Lobo RA. Evaluation of cardiovascular event rates with hormone therapy in healthy, early postmenopausal women: results from 2 large clinical trials. Arch Intern Med. 2004; 164(5):482-4.

15. Rossouw JE, Prentice RL, Manson JE, Wu L, Barad D, Barnabei VM, et al. Postmenopausal hormone therapy and risk of cardiovascular disease by age and years since menopause. JAMA. 2007;297(13):1465-77.

16. Manson JE, Allison MA, Rossouw JE, Carr JJ, Langer RD, Hsia, J, et al. Estrogen therapy and coronary-artery calcification. N Engl J Med. 2007;356(25):2591-602.

17. Anderson TJ, Uehata A, Gerhard MD, Meredith IT, Knab S, Delagrange D, et al. Close relation of endothelial function in the human coronary and peripheral circulations. J Am Coll Cardiol. 1995;26(5):1235-41.

18. Fernandes CE, Pinho-Neto JSL, Gebara OCE, Santos Filho RD, Pinto Neto AM, Pereira Filho AS, et al. I Diretriz Brasileira sobre prevenção de DCV em mulheres climatéricas e a influência da Terapia de Reposição Hormonal (TRH) da Sociedade Brasileira de Cardiologia (SBC) e da Associação Brasileira do Climatério (SOBRAC). Arq Bras Cardiol. 2008;91 (1 Supl 1):1-23. 